\title{
BIÓPSIA ENDOMIOCÁRDICA DE PACIENTES SUBMETIDOS A TRANSPLANTE CARDÍACO: ESTUDO DESCRITIVO DO PROCEDIMENTO E ANÁLISE HISTOLÓGICA DOS FRAGMENTOS
}

\author{
Endomyocardial biopsy of patients submitted to heart transplantation: \\ descriptive study and histologic analysis of the specimens
}

\begin{abstract}
Josué V. Castro Neto ${ }^{1}$, Alexandre R. de Carvalho ${ }^{2}$, Carlos Mendez Contreras ${ }^{2}$, Reginaldo Cipullo ${ }^{3}$, Marco Aurélio Finger $^{3}$, Paulo Chaccur ${ }^{2}$, Mabel Zamorano ${ }^{4}$, Ricardo Manrique ${ }^{5}$, Hélio M. Magalhães ${ }^{6}$, Jarbas Jackson Dinkhuysen ${ }^{7}$
\end{abstract}

\begin{abstract}
RESUMO
Objetivo: descrever a técnica empregada, as complicações e as alterações histológicas de pacientes submetidos à biópsia endomiocárdica no pós-operatório de transplante cardíaco. Métodos: estudo transversal de 232 procedimentos de biópsia endomiocárdica do ventrículo direito em 38 pacientes que foram submetidos a transplante cardíaco entre janeiro de 2000 e dezembro de 2002 . Os critérios de exclusão foram: óbitos hospitalares (sem realização de biópsia) e dados incompletos para preenchimento de protocolo. Resultados: A técnica empregada foi a da punção da veia jugular interna direita. O biótomo utilizado foi o de $2,2 \times 510 \mathrm{~mm}$ e a localização de sua ponta confirmada por fluoroscopia. Obtiveram-se, em média, 5,1 fragmentos por biópsia. Dez biópsias $(4,3 \%)$ não foram interpretadas. Em 42 procedimentos $(18,1 \%)$ não foi evidenciada rejeição (grau 0), segundo a classificação da sociedade internacional. Sendo que entre as rejeições, a mais comum foi a aguda, focal e discreta (grau IA) - 55\%. Até seis meses após o implante, o grau IA foi de $41,2 \%$ e o grau IB (difusa e discreta) de $32,4 \%$. A partir do sexto mês, os graus foram de 56\% e 12,5\%, respectivamente. Ocorreram nove complicações (3,8\%). A mais comum foi o hemopericárdio com necessidade de drenagem pericárdica $(1,3 \%)$. Conclusões: A biópsia endomiocárdica do ventrículo direito foi um procedimento realizado com baixa morbidade, sendo a principal complicação o hemopericárdio. Pela avaliação do grau histológico de rejeição aguda, o principal tipo foi o grau IA, sendo 41,2\% até seis meses e $56 \%$ após seis meses do transplante cardíaco.
\end{abstract}

Descritores: Procedimentos cirúrgicos cardiovasculares, Transplante, Rejeição celular, Transplante cardíaco, Biópsia endomiocárdica.

\footnotetext{
Trabalho realizado no Instituto Dante Pazzanese de Cardiologia (IDPC) - Av. Dante Pazzanese, 500 - São Paulo - SP - Brasil.

1. Doutor em Cirurgia Torácica e Cardiovascular FMUSP;

2. Especialista em Cirurgia Cardiovascular;

3. Especialista em cardiologia - médico da Seção de Transplante Cardíaco - IDPC;

4. Especialista em patologia - médica do IDPC;

5. Especialista em imunohematologia - médico da Seção de Transplante Cardíaco - IDPC;

6. Professor Livre-Docente em Cardiologia - FMUFG;

7. Professor Livre-Docente em Cirurgia Torácica e Cardiovascular - FMUSP e Chefe da Seção de Transplante Cardíaco - IDPC.

Endereço para correspondência: Josué V. Castro Neto - Rua Rio Grande, 321/142 CEP: 04018-000 - São Paulo - SP - Fone: (85) 8724-2450 - E-mail: jvcn@uol.com.br

Recebido em: 30.06 .2003 Aceito em: 20.01.2005
}

\section{INTRODUÇÃO}

A biópsia endomiocárdica com avaliação histológica dos fragmentos biopsiados permanece como método padrão para detecção da rejeição após transplante cardíaco. Em 1962, foi demonstrada a técnica do procedimento, além do desenvolvimento de um biótomo específico. ${ }^{1}$ Dez anos após, foi aperfeiçoado o biótomo, criando-se o instrumento que se utiliza até os dias de hoje: o biótomo de Caves-Stanford. ${ }^{2}$

Outro grande passo nesse sentido foi dado com a uniformização da graduação histológica da rejeição do enxerto. ${ }^{3}$ Assim, os resultados apresentados por diversos grupos puderam ser melhor interpretados. Este trabalho objetiva descrever o procedimento que é realizado no Instituto e analisar os resultados histológicos dos fragmentos obtidos pela biópsia endomiocárdica (BEM) do ventrículo direito (VD) no pós-operatório de transplante cardíaco.

\section{CASUÍSTICA E MÉTODO}

Entre janeiro de 2000 e dezembro de 2002, 53 pacientes foram submetidos a transplante cardíaco. Entre esses, selecionaram-se 38 pacientes. Foi realizado um estudo transversal dos resultados histológicos dos fragmentos obtidos da BEM do VD. Os critérios de 
exclusão foram: óbitos hospitalares nos quais não se realizou BEM e dados incompletos para preenchimento de protocolo.

A protocolo de realização de BEM no serviço é a seguinte: primeira biópsia ao redor do décimo dia de pós-operatório e mais duas biópsias semanais no primeiro mês; no segundo e terceiro meses, quinzenalmente; no quarto, quinto e sexto meses, mensalmente; a partir do sexto mês, semestralmente e após o primeiro ano, anualmente.

O procedimento é realizado via ambulatorial, entrando o paciente no centro cirúrgico pela manhã e permanecendo em observação por seis horas, quando são realizados eletrocardiograma e radiografia de tórax. O material utilizado para realização do procedimento consiste em: seringas, agulhas, introdutor percutâneo 7f, biótomo tipo MKA-6 2,2 x 510 mm (Fehling surgical instruments). Sob anestesia local e eventual sedação, por punção da veia jugular interna direita, introduzimos bainha 7f. Através dessa, o biótomo é posicionado no VD através de fluoroscopia (figura 1). São obtidos os fragmentos endomiocárdicos. Após isso, é retirado o introdutor e realizada compressão hemostática.

Os fragmentos são enviados para estudo histológico em solução contendo formalina a $10 \%$. São desidratados com álcool a 95\% e absoluto, diafanizados com xilol e preparados para corte com parafina. As lâminas são coradas com hematoxilina-eosina e tricrômio de Masson para estudo com microscopia óptica.

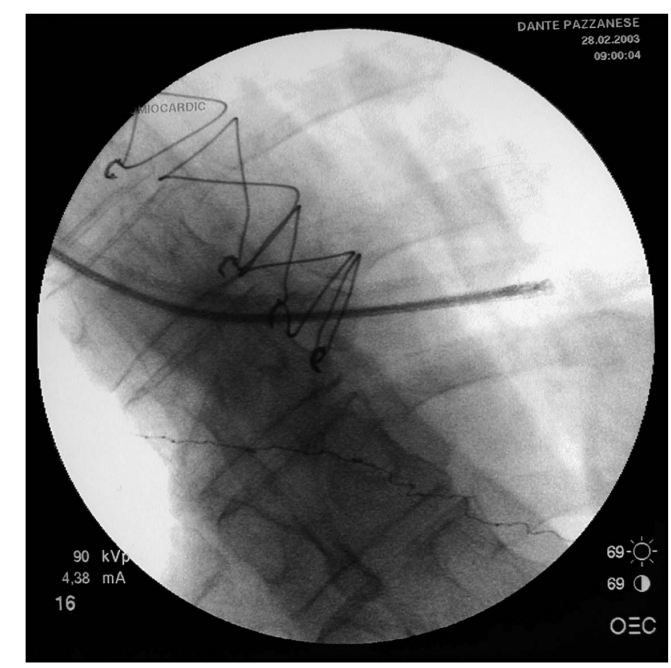

\section{RESULTADOS}

Foram realizados 232 procedimentos em 38 pacientes $(6,1$ procedimentos por paciente) no período estudado, sendo que em cada procedimento obteve-se, em média, 5,1 fragmentos. Dez biópsias $(4,3 \%)$ não foram interpretadas. Duzentas e vinte e duas biópsias foram estudadas. Segundo a classificação padronizada de Billingham, 42 biópsias (18,1\%) foram grau 0. Cento e oitenta biópsias variaram de graus I a IIIB. O efeito quilty esteve presente em 96 biópsias, sendo tipo A em 68 e tipo B em 28. Já rejeição humoral foi evidenciada em 59 oportunidades. O número de biópsias por grau histológico foi de: Grau IA - 99, Grau IB - 64, Grau II -12, Grau IIIA -3, Grau IIIB -2, Grau IV-0 (gráfico 1).

O grau I foi o mais prevalente (163 resultados), sendo o IA responsável por $55 \%$ das biópsias interpretadas.

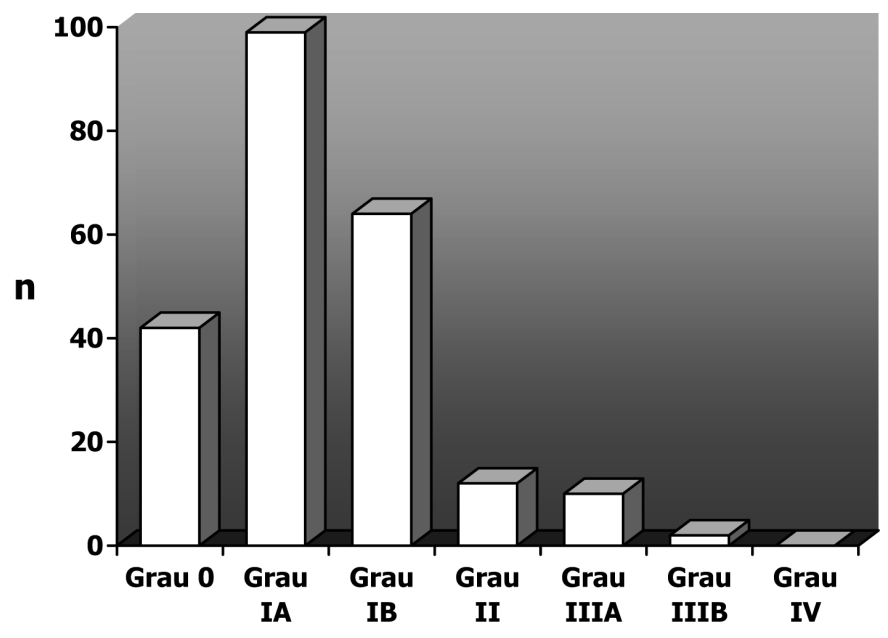

Gráfico1. Número de biópsias de acordo com o grau de rejeição histológica em 222 procedimentos realizados em 38 pacientes no pós-operatório de transplante cardíaco.

Dividiram-se as biópsias interpretadas por período realizado: até seis meses e após seis meses do transplante cardíaco. No primeiro período foram realizados 182 procedimentos, enquanto que no segundo período, 40 procedimentos.

Entre os seis primeiros meses os graus histológicos foram assim divididos: Grau 0 - 34 (18,6\%), Grau IA - 75 (41,2\%), Grau IB - 59 $(32,4 \%)$, Grau II -10 (5,5\%), Grau IIIA -2 (1,1\%), Grau IIIB -2 $(1,1 \%)$, Grau IV-0.

Após os seis primeiros meses, a divisão foi assim: Grau $0-8(20 \%)$, Grau IA - 24 (56\%), Grau IB - 5 (12,5\%), Grau II -2 (5\%), Grau IIIA -1 (2,5\%), Grau IIIB -0, Grau IV-0 (gráfico 2).

As complicações estiveram presentes em nove procedimentos $(3,8 \%)$. A mais comum foi o hemopericárdio que ocorreu em três pacientes $(1,9 \%)$. Nesses três casos foi necessária drenagem pericárdica. Em dois casos ocorreu arritmia sem repercussão hemodinâmica. Pneumotórax, irritação neural, hematoma venoso local e punção carotídea em um caso de cada.

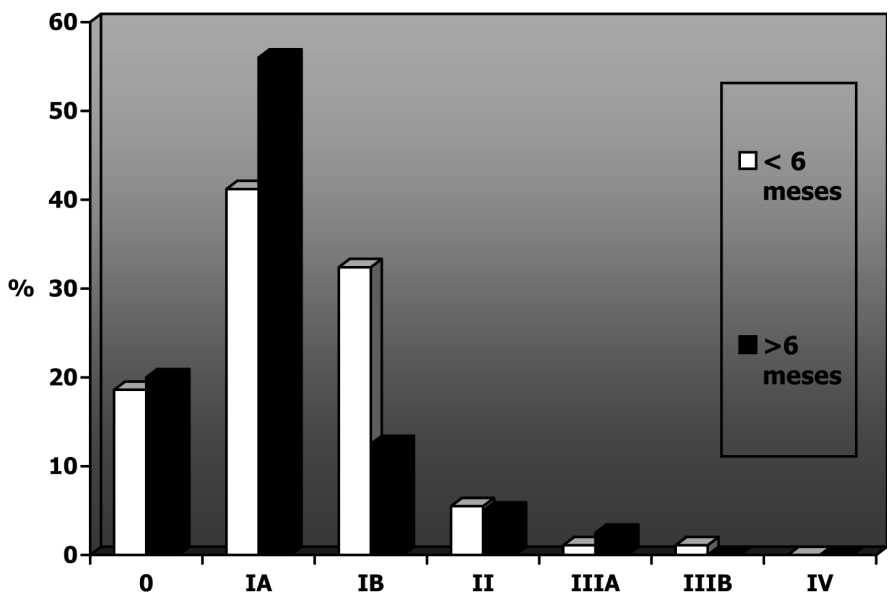

Gráfico 2. Graduação Histológica de 222 Biópsias Endomiocárdicas segundo o tempo de realização do procedimento. Antes de 6 meses após transplante $(n=182)$ e após 6 meses $(n=40)$. 


\section{DISCUSSÃO}

Desde as primeiras descrições de tentativas de biópsia do coração transplantado, ${ }^{1,2,4}$ a técnica de obtenção de fragmentos do miocárdio evoluiu significativamente. Hoje, o procedimento pode ser realizado com baixa morbidade e via ambulatorial.

No Instituto Dante Pazzanese de Cardiologia, o procedimento é realizado preferencialmente pelo acesso venoso do pescoço através da punção da veia jugular interna direita. Entretanto, outros sítios possíveis são as veias femoral e subclávia e a artéria femoral. ${ }^{5}$

Outro grande avanço foi a padronização da graduação histológica da rejeição aguda celular do enxerto cardíaco. ${ }^{3}$ Essa classificação (da sociedade internacional) permite o diagnóstico da ausência ou presença de rejeição celular em sete estágios.

Foram estudados os procedimentos de BEM do VD em 38 pacientes submetidos a transplante cardíaco ortotópico, pela técnica bicaval e bipulmonar. Enfatizou-se a avaliação da graduação histológica e das complicações do procedimento.

Foram realizadas 232 BEM do VD, perfazendo um total de 6,1 procedimentos por paciente no intervalo do estudo.

Em cada procedimento obtiveram-se, em média, 5,1 fragmentos. Esse número parece satisfatório, pois o ideal é mais que quatro fragmentos com pelo menos $50 \%$ desses sendo miocárdio., ${ }^{3,6}$ As dez biópsias $(4,3 \%)$ não interpretadas foram devido à amostra inadequada.

Estudaram-se, portanto, 222 biópsias. Segundo a classificação padronizada de Billingham, em 42 biópsias $(18,1 \%)$ o grau foi 0 . As outras biópsias (180) variaram entre graus I e IIIB.

O efeito quilty esteve presente em 96 biópsias, sendo tipo A (sem envolvimento miocitário) em 68 e tipo B (com envolvimento miocitário) em 28. Controvérsia existe se esse efeito, que consiste em infiltrado linfocitário endocárdico, é benigno ou pode predizer rejeição. ${ }^{7}$ Já rejeição humoral foi evidenciada em 59 ocasiões.

A prevalência da graduação histológica em cada grupo foi: Grau IA - 99, Grau IB - 64, Grau II -12, Grau IIIA -3, Grau IIIB -2, Grau IV-0. A rejeição celular aguda, focal e discreta (IA), que traduz infiltração de grandes linfócitos perivasculares sem dano miocitário, foi a mais comum (55\%).
Quando se dividiram as biópsias realizadas em dois períodos, até seis meses e após seis meses, verificou-se que a grande maioria foi realizada até os seis meses ( $\mathrm{n}=182$ procedimentos). Isso era esperado pelo fato de que em nosso protocolo o número de biópsias decresce ao longo do tempo de tal maneira que, após seis meses, as biópsias passam a ser realizadas semestralmente e anualmente; o estudo envolveu os pacientes operados entre 2000 e 2002, respeitando os critérios de exclusão.

Entre os seis primeiros meses $(\mathrm{n}=182)$, o número de biópsias em cada grau foi de: Grau 0 - 34 (18,6\%), Grau IA - $75(41,2 \%)$, Grau IB - 59 (32,4\%), Grau II -10 (5,5\%), Grau IIIA -2 (1,1\%), Grau IIIB -2 (1,1\%), Grau IV-0.

Após os seis meses ( $\mathrm{n}=40)$, a divisão foi assim: Grau $0-8(20 \%)$, Grau IA - 24(56\%), Grau IB - 5(12,5\%), Grau II -2(5\%), Grau IIIA -1(2,5\%), Grau IIIB -0, Grau IV-0(gráfico 2).

No primeiro semestre, o percentual de resultados de Grau IA e IB foi de $41,2 \%$ e de $32,4 \%$, respectivamente. Já após o primeiro semestre, o percentual foi de $56 \%$ e $12,5 \%$, respectivamente. Além do que, ocorreram dois casos de rejeição tipo IIIB no primeiro e nenhum caso no segundo.

Há concordância na literatura de que os episódios de rejeição decrescem com o tempo. Os dados apresentados, apesar de não avaliados por teste estatístico, mostram percentual menor de grau IB após seis meses de transplante.

Por fim, nosso índice de complicações foi maior que o demonstrado na literatura. ${ }^{8}$ Entretanto, se desconsiderarmos os dois casos de arritmia transitória sem repercussão hemodinâmica e o caso de hematoma venoso local, esse índice cairia para menos de $2 \%$. Número ainda um pouco alto, mas próximo ao reportado por outros autores.

\section{CONCLUSÃO}

A biópsia endomiocárdica do ventrículo direito, empregando a técnica de punção da veia jugular interna direita, foi um procedimento realizado com baixa morbidade, sendo a principal complicação o hemopericárdio. Pela avaliação do grau histológico de rejeição aguda, o principal tipo foi o grau IA, sendo $41,2 \%$ até seis meses e $56 \%$ após seis meses do transplante cardíaco.

\section{ABSTRACT}

Objective: to describe the technique, complications and histologic alterations of patients submitted to endomyocardial biopsy in the post-operative period of heart transplantation. Methods: we study 232 procedures of right ventricle endomyocardial biopsy of 38 patients operated between January 2000 and December 2002. Exclusion criteria were: in hospital mortality (not submitted to biopsy) and incomplete criteria for protocol. Results: all procedures were done through the right internal jugular vein. The bioptome utilized was a $2,2 \times 510 \mathrm{~mm}$ - 7Fr. These were done under fluoroscopic guidance. In average we obtain 5,1 pieces by procedure. In $48(18,1 \%)$ procedures no cellular rejection (grade 0) was evidenced. Between rejections, the most common was acute, focal and mild (grade IA)-55\%. In the 6 months period after surgery, grade IA was $41,2 \%$ and grade IB (diffuse and mild) $32,4 \%$. After 6 months, were $56 \%$ and $12,5 \%$, respectively. There were 9 complications $(3,8 \%)$. The most common was hemopericardium with pericardial drainage (1,3\%). Conclusions: Right ventricle endomyocardial biopsy was accomplished with low morbidity. The principal complication was hemopericardium. Avaliation of standardized histologic grading revealed that the principal type was grade IA and that in the 6 months period after surgery it was $41,2 \%$ and after 6 months it was $56 \%$.

Key words: Cardiovascular surgical procedures, Transplant, Celular rejection, Heart transplantation, Endomyocardial biopsy. 


\section{REFERÊNCIAS}

1. Sakakibara S, Konno S. Endomyocardial biopsy. J Dis Chest 1963; 44:345-350.

2. Caves PK, Stinson EB, Billingham M, Shumway NE. Percutaneos transvenous endomyocardial biopsy in human hearts recipients - experience with a new technique. Ann Thorac Surg 1973; 16(4):325-335

3. Billingham ME, Cary NRB, Hammond ME, Kemnitz J, Marboe C, McCallister HA et al. A working formulation for the standardization of nomenclature in the diagnosis of heart and lung rejection. J Heart Lung Transplant 1990;9:587-593.

4. Shirey EK, Hawk WA, Mukerji D, Effler DB. Percutaneos myocardial biopsy of the left ventricle: experience in 198 patients. Circulation 1972; 46:112-122.
5. Anderson JL, Marshall HW. The femoral venous approach to endomyocardial biopsy: comparison with internal jugular and transarterial aproaches. Am J Cardiol 1984;53:833-837.

6. Bourge RC, Rodriguez ER, Tan CD. Cardiac allograft rejection. In:Kirklin JK, YoungJB,McGiffinDC(eds).Hearttransplantation.ChurchillLivingstone;2002. pp464-520.

7. Luthringer DJ, Yamashita JT, Czer LS, Trento A, Fishbein MC. Nature and significance of epicardial lymphoid infiltrates in cardiac allografts. J Heart Lung Transplant 1995;14:537-543.

8. Bhat G, Burwig S, Walsh R. Morbidity of endomyocardial biopsy in cardiac transplant recipients. Am Heart J 1993;125:1180-1181. 